\title{
Effects of Nonuniform Fiber Sensitivity, Innervation Geometry, and Noise on Information Relayed by a Population of Slowly Adapting Type I Primary Afferents from the Fingerpad
}

\author{
Antony W. Goodwin and Heather E. Wheat \\ Department of Anatomy and Cell Biology, University of Melbourne, Parkville, Victoria 3052, Australia
}

The capacity of a population of primary afferent fibers to signal information about a sphere indenting the fingerpad is limited by factors such as the inhomogeneity of sensitivity among the afferents, the pattern and density of innervation, and the effects of noise (response variability). Using experimental data recorded from single slowly adapting type I afferents (SAls), we simulated the response of the SAI population to such a stimulus. The human ability to discriminate stimulus curvature, location, and force has been quantified previously. We devised three neural measures, treating them as surrogates for the real neural measures underlying human performance, and explored how population parameters usually overlooked in neural coding studies affect such measures. Variation in sensitivity among SAls is large; this distorts population response profiles markedly but has no significant impact on the neural measures. Two classes of noise were introduced, one dependent on and the other independent of the level of neural activity. Resolution of the model was compared with discrimination in humans. Correlation of noise among neurons had different effects for the different measures. An increase in correlation decreased resolution in the measure for force but improved resolution in the measure for position. Increasing innervation density (1) always increased resolution for position and (2) increased resolution for force if noise was uncorrelated but had diminishing effects as correlation increased. Correlation and innervation density had complex effects on the measure for curvature, depending on the class of noise. Nonuniformity in the pattern of innervation had negligible effects on resolution.

Key words: tactile resolution; population response; neural code; innervation density; neural noise; correlated noise; covariance; tactile shape; position on skin; contact force
Responses of single peripheral nerve fibers innervating the fingerpads have been characterized for stimuli similar to objects that humans manipulate everyday with precision. The multiple parameters of such objects, such as their shape, orientation with respect to the fingers, or position on the skin, cannot be resolved by single units but are represented clearly in the responses of whole populations of fibers (LaMotte and Srinivasan, 1987a,b, 1996; Srinivasan and LaMotte, 1987; Ray and Doetsch, 1990; Cohen and Vierck, 1993; LaMotte et al., 1994; Khalsa et al., 1998). There are a number of characteristics inherent in such populations that limit their capacity to signal information. For example, it has been evident for a long time that the afferents are inhomogeneous, varying widely in their sensitivity (Knibestol, 1975), and that innervation density varies from region to region (Johansson and Vallbo, 1979). However, there have been no quantitative analyses of the limitations imposed by such factors.

After the initial focus on single neurons (Barlow, 1972), it soon became obvious that even relatively simple aspects of perception, such as determining the intensity of a vibratory probe on the skin, could not be explained by the properties of single cells considered in isolation (Johnson, 1974). For more complex aspects of perception, the neural bases were only evident when ensembles of neurons were examined (Georgopoulos et al., 1986; Gochin et al., 1994). Further progress has been limited by technology; although

\footnotetext{
Received Feb. 25, 1999; revised June 21, 1999; accepted June 24, 1999.

This work was supported by a grant from the National Health and Medical Research Council of Australia.

Correspondence should be addressed to A.W. Goodwin, Department of Anatomy and Cell Biology, University of Melbourne, Parkville, Victoria 3052, Australia.

Copyright (C) 1999 Society for Neuroscience 0270-6474/99/198057-14\$05.00/0
}

it is possible to record simultaneously from several neurons, the number is only a small fraction of the active population (Aertsen et al., 1991; Mountcastle et al., 1991; Lee et al., 1998). Thus, to characterize processing in ensembles of neurons, less direct methods are required.

A number of different approaches have been used. First, general theoretical studies (Johnson, 1980b; Gerstein and Aertsen, 1985; Fetz, 1997; Rieke et al., 1997) have provided a framework for analyzing neural populations highlighting, among other issues, the enhancement of signal-to-noise ratios in ensembles of neurons (Zohary et al., 1994). Second, neural network modeling has been invaluable in emphasizing the distributed nature of population coding (Lehky and Sejnowski, 1990; Robinson, 1992). In the third approach, data from single-cell recordings have been used to simulate realistic whole populations of neurons, allowing an investigation of the effects of factors, such as the number of active neurons (Paradiso, 1988; Shadlen et al., 1996; Zhang et al., 1998).

In previous studies, we recorded from slowly adapting type I primary afferents (SAIs) when spherical stimuli were applied passively to the fingerpad and developed a mathematical description of those responses (Goodwin et al., 1995, 1997; Wheat et al., 1995). In matching psychophysics experiments, we quantified the human capacity to scale and discriminate three parameters of the stimulus: its curvature, position on the skin, and contact force (Goodwin et al., 1991). In the current study, we build on those single-fiber data by simulating the whole population response. Neural measures for the three stimulus parameters are extracted and compared with human performance. This approach allows us to answer specific questions about the inherent properties of the 
A

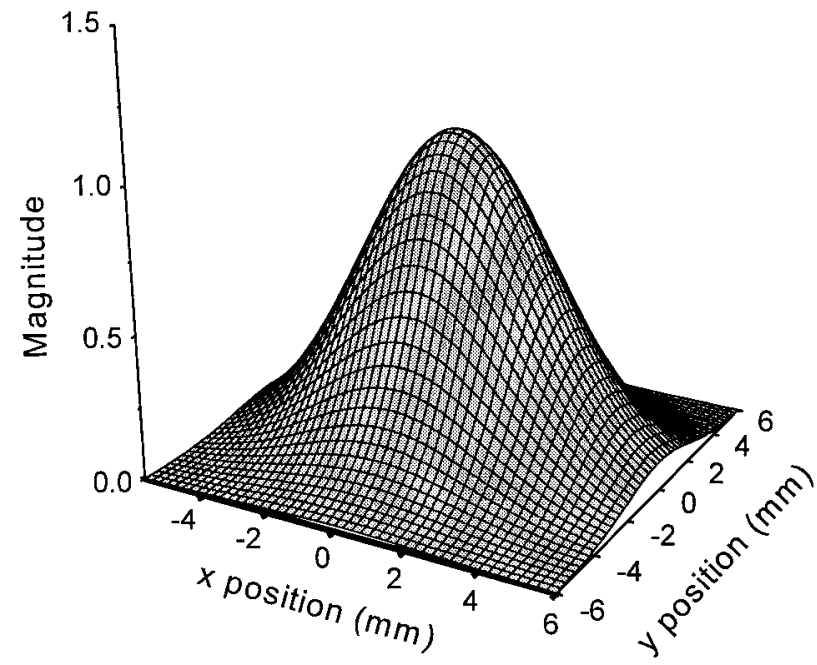

B

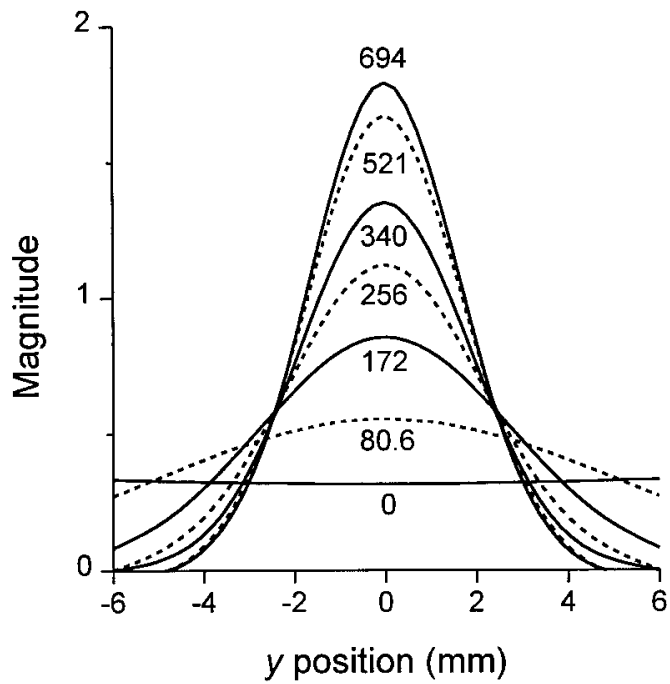

Figure 1. Gaussian curves of the form $a e^{-\left(b x^{2}+c y^{2}\right)}$ reflecting the "receptive field profile" common to all SAIs on the monkey fingerpad. $A$, Profile shown for both directions on the finger for a sphere of curvature $256 \mathrm{~m}^{-1}$. B. Profiles along the $y$-axis $(\mathrm{x}=0)$ for spheres with curvature $694,521,340,256$, $172,80.6$, and $0 \mathrm{~m}^{-1}$. These profiles may also be viewed as the responses of an ideal population of SAIs, all with the same sensitivity, when a sphere is located at the origin.

population, such as the following. How does the variation in sensitivity among afferents compromise stimulus representations? How does the innervation density affect the resolution of shape? Is the geometry of innervation important? How are different neural measures affected by response variability? Our aim is to elucidate how the population characteristics affect the different types of neural measures rather than to accept or reject specific candidate codes.

\section{MATERIALS AND METHODS}

Background. Reconstructions presented in this paper were derived from data recorded from 55 SAIs innervating the fingerpads of Macaca nemestrina monkeys (Goodwin et al., 1995; Wheat et al., 1995). The receptive fields of the afferents were located on the central, relatively flat, portion of the finger. When a sphere was applied to the skin, it was found that the response of any SAI could be expressed as the product of two factors: one determined by the curvature of the sphere and the position of the receptive field relative to the sphere, and the second representing the sensitivity of the afferent. Regression of the data showed that the first factor could be described by a two-dimensional Gaussian function. Thus, for any SAI, the response $r$ was given by:

$$
r=s a \mathrm{e}^{-\left(b x^{2}+c y^{2}\right)}
$$

where the afferent, with sensitivity $s$, had a receptive field center located at coordinates $x$ and $y$ (distances from the point where the sphere first contacted the skin in directions orthogonal to and parallel to the axis of the finger, respectively). Values of the constants $a, b$, and $c$ were determined by the curvature of the sphere and were the same for all afferents; values for these constants can be found in Goodwin et al. (1995). The Gaussian profiles, which are central to our simulation, are illustrated in Figure 1. Changing the contact force scaled the profiles by a multiplicative constant.

The simulation. Our aim is to simulate the activity of populations of SAIs in the human fingerpad when a sphere, of variable curvature, contacts the skin. In the model, the receptive field centers of the fibers are located at discrete positions $\left(x_{\mathrm{i}}, y_{\mathrm{j}}\right)$ in a matrix on the skin (Fig. 2). For most of the simulations, the receptive field centers are uniformly spaced, with the same spacing $(\delta \mathrm{mm})$ in the $x$ and $y$ directions. The point of initial contact between the sphere and the skin is located at the origin of the matrix. The total area spanned by the receptive field centers is kept constant at $13.2 \times 13.2 \mathrm{~mm}$, which is consistent with the size of human
A

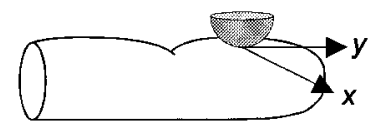

B

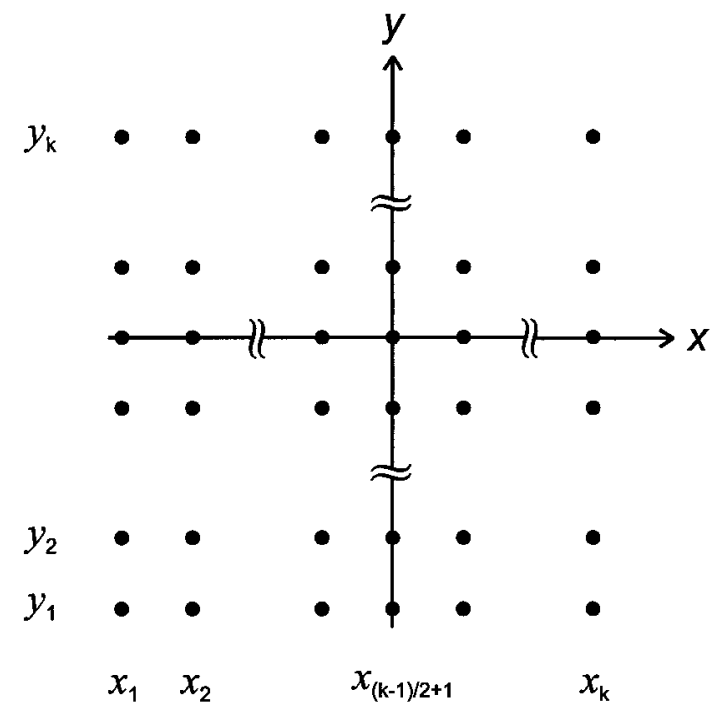

Figure 2. Geometry of the simulation. $A$, The sphere is located centrally on the fingerpad with the $x$ - and $y$-axes passing through the point of initial contact. $B$, Receptive field centers of afferents are located on the skin at points in a matrix with positions $x_{\mathrm{i}}$ and $y_{\mathrm{j}}$. For most simulations, $i=1, k$ and $j=1, k$ and $k$ is odd so that a receptive field center is located at the origin, which corresponds to $x_{(\mathrm{k}-1) / 2+1}, y_{(\mathrm{k}-1) / 2+1}$. For uniform innervation density, the distance between receptive field centers is the same in the $x$ and $y$ directions $(\delta)$.

fingerpads. There is a fixed relationship $n \delta^{2}=13.2 \times 13.2$ between the total number of fibers $n$, the distance between receptive field centers $\delta$, and the $13.2 \times 13.2 \mathrm{~mm}$ innervated region of skin; the innervation density is given by $1 / \delta^{2} \mathrm{~mm}^{-2}$. Initially, the spacing is set to $1.2 \mathrm{~mm}$, which 
corresponds to an innervation density of $0.7 \mathrm{~mm}^{-2}$, the value estimated by Johansson and Vallbo (1979).

We have assumed that the underlying Gaussian receptive field profiles in humans are the same as those measured by us in monkeys. The basis for this assumption is that the active population is on the relatively flat portion of the finger in which skin and receptor mechanics are likely to be similar in humans and monkeys. Our limited measurements of profiles in human peripheral nerves corroborate this (Goodwin et al., 1997). For each afferent in the model, the response depends on three factors. The first factor comprises the stimulus parameters (the curvature and position of the sphere and the contact force), and these are reflected in the Gaussian profile. The second factor is the sensitivity of the fiber. Later in the manuscript, we will introduce the third factor, which is noise affecting the response of the fiber and also the subsequent processing of that response by the CNS. Thus, in the absence of noise, the response of any afferent is given by:

$$
r_{\mathrm{ij}}=s_{\mathrm{ij}} k a e^{-\left(b x_{\mathrm{i}}^{2}+c y_{\mathrm{j}}^{2}\right)}
$$

In the above equation, the terms are defined as follows. The afferent with a receptive field center located in the matrix (Fig. 2) at position $\left(x_{\mathrm{i}}, y_{\mathrm{j}}\right)$ has a response $r_{\mathrm{ij}}$ measured by the number of impulses occurring in the first second of response (imp s${ }^{-1}$ ). This is the time period that was used in the analysis of our experimental data from monkeys and humans. The sensitivity $s_{\mathrm{ij}}$ of each fiber in the matrix varies randomly from fiber to fiber; the distribution of $s$, derived from our experimental data, is normal with a mean \pm SD of $40 \pm 15.5$ (Goodwin et al., 1995). Changing the curvature of the sphere changes the values of the constants $a, b$, and $c$. In our neural experiments, we estimated these constants from data at seven different curvatures. Here, we use those seven sets of values; for curvatures in between, the constants are determined by interpolation. Contact force is set by the constant $k$, which was also estimated experimentally.

Population response measures. A number of measures is calculated from each simulated population response. These measures were chosen as being plausible representations of the position, curvature, and contact force of the stimulus and are detailed in Results.

Discrimination performance. Estimates of the difference limen are used to assess the ability of the model to discriminate two stimuli. A twoalternative forced-choice paradigm equivalent to that used in our human psychophysics experiments is used. Standard signal detection theory is used to calculate an unbiased measure of discrimination $d^{\prime}$ as follows. Two stimuli differing in one parameter, the standard $S$ and the comparison $C$, are presented to the model in pairs. For 100 pairs, the first and second stimuli are both the same and are both the standard $\left(S_{1}, S_{2}\right)$, and for 100 pairs, the first stimulus is the standard and the second is the comparison $\left(S_{1}, C_{2}\right)$. From the responses of the afferents to the standard stimulus, a code or measure for the relevant stimulus parameter is extracted as $m^{\mathrm{s}}$ so that the pair $S_{1}, S_{2}$ result in a pair of measures $m_{1}^{\mathrm{s}}, m_{2}^{\mathrm{s}}$. Similarly, a pair of stimuli $S_{1}, C_{2}$ result in a pair of measures $m_{1}^{\mathrm{s}}, m_{2}^{\mathrm{c}}$. In the presence of noise, each response is different, even if the stimulus does not change.

A decision boundary bnd is defined by half of the difference between the mean value of the measure for the comparison stimulus and the mean value for the standard stimulus. Thus,

$$
\text { bnd }=0.005\left|\sum_{100} m_{2}^{\mathrm{c}}-\sum_{100} m_{1}^{\mathrm{s}}\right|
$$

Because there is no interaction between successive stimuli in the model, the mean of $m_{2}^{\mathrm{s}}$ is identical to the mean of $m_{1}^{\mathrm{s}}$.

For each pair $S_{1}, S_{2}$, the stimuli are judged by the model to be different if $\left|m_{2}^{\mathrm{s}}-m_{1}^{\mathrm{s}}\right|$, and the stimuli for pair $S_{1}, C_{2}$ are judged different if $\mid m_{2}^{\mathrm{c}}-$ $m_{1}^{\mathrm{s}} \mid \geq$ bnd; otherwise, the two stimuli are judged to be the same. From the conditional probabilities $\mathrm{P}$ (judged different $244 S S$ ) and $\mathrm{P}$ (judged different $244 S C$ ), $d^{\prime}$ is calculated. Using a range of comparison stimuli, $d^{\prime}$ is plotted against the difference between the comparison and standard stimuli, and linear regression is used to obtain the difference limen corresponding to a $d^{\prime}$ value of 1.35 (Johnson, 1980a; Goodwin et al., 1991).

Stimulus parameter values. When characterizing the curvature or position of the stimulus, a contact force of $147 \mathrm{mN}$ [15 grams force (gf)] is used; this is the force used by us in our monkey experiments. A number of studies in which corresponding tactile stimuli have been used in monkeys and humans indicate that a force of $147 \mathrm{mN}$ in monkeys is equivalent to the force of $490 \mathrm{mN}$ (50 gf) used by us in human experiments; for example, the afferent responses are comparable in the two species (Goodwin et al., 1997). When characterizing curvature or contact force, the stimulus is located at the center of the innervated region of skin. The values of the standard stimuli used to characterize discrimination performance of the model and the ranges of values used to characterize scaling performance are the same as those used in our human psychophysics experiments.

The simulation is written in Fortran, and random variables, all of which have a Gaussian distribution, and are generated using the algorithms of Press et al. (1986).

\section{RESULTS}

\section{Population response measures}

Parameters of the population, such as innervation density, are major factors in determining the characteristics of the neural representation of a stimulus. To quantify these effects, a population response measure was calculated for each of the three stimulus parameters that we vary (curvature, location, and force). The measures are used to indicate similarities and differences in the way real neural codes for the three stimulus parameters would be affected by the population parameters.

The rationale for the measures is developed from the curves in Figure 1, which can be viewed as the responses of an ideal population in which all afferents have the same sensitivity and there is no noise. When the position of the sphere on the finger shifts, there is a corresponding shift of the response profile within the afferent population. Thus, the position of the stimulus is clearly signaled by the locus of the center of neural activity, which is simply calculated by the $x$ and $y$ components of the centroid of the three-dimensional profile:

$$
x_{\mathrm{cent}}=\frac{\sum_{i, j} x_{\mathrm{i}} r_{\mathrm{ij}}}{\sum_{i, j} r_{\mathrm{ij}}} \text { and } y_{\mathrm{cent}}=\frac{\sum_{i, j} y_{\mathrm{j}} r_{\mathrm{ij}}}{\sum_{i, j} r_{\mathrm{ij}}}
$$

The solid line in Figure $3 A$ shows the $y$ component of the centroid as a function of the $y$ position of the stimulus. This curve matches the human scaling function for position documented by Goodwin and Wheat (1999).

As the sphere increases in curvature, the response profiles in Figure $1 B$ become higher and narrower. For the flat surface (curvature $0 \mathrm{~m}^{-1}$ ), the response of each afferent equals the mean response of all the afferents. As the curvature of the sphere increases, responses of individual afferents deviate further from the mean response of all afferents. Thus, a simple measure of the curvature is the second moment of the responses about the mean. The calculation, normalized for independence of innervation density and response magnitude, is given by:

$$
\text { secmom }=\frac{\sum_{i, j}\left(r_{\mathrm{ij}}-\bar{r}\right)^{2}}{\delta^{2}\left(\sum_{i, j} r_{\mathrm{ij}}\right)^{2}} \text { where the mean } \bar{r}=\frac{\sum_{i, j} r_{\mathrm{ij}}}{n}
$$

The solid line in Figure $3 B$ shows this measure as a function of the curvature of the stimulus; the function is remarkably similar to the human scaling function for perceived curvature published by Goodwin et al. (1991) showing a tendency to saturate at higher curvatures. The independence of the measures is illustrated by the crosses in Figure $3 B$, which show that changes in curvature do not affect the centroid, which is our measure for the position of the stimulus.

Increasing the contact force scales the response profiles in Figure $1 B$ upward (Goodwin et al., 1995) so that any measure of overall activity will increase with increasing force. Perhaps the simplest measure is the sum of the responses of the afferents. The sum certainly increases with an increase in contact force, but 
A. Position

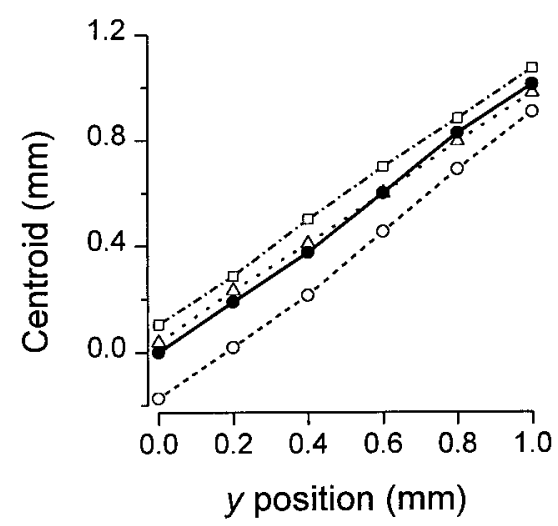

B. Curvature

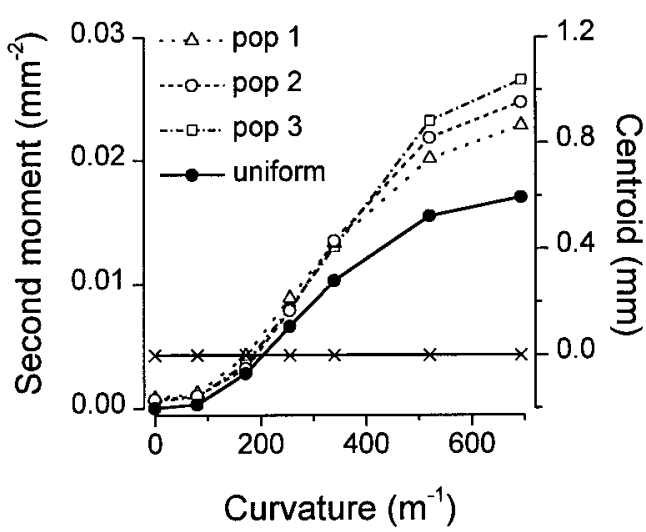

\section{Force}

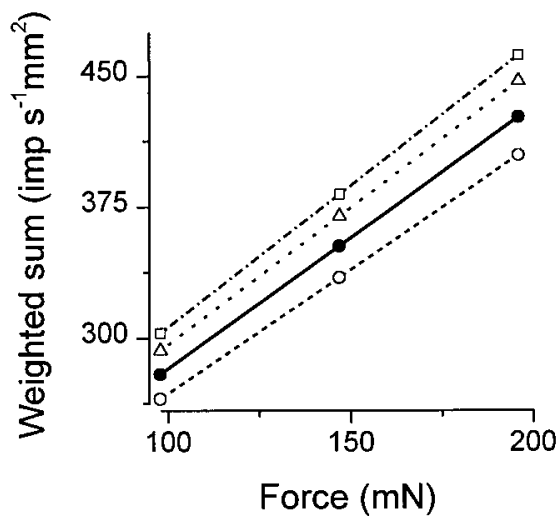

Figure 3. Proposed population measures for the position, curvature, and contact force of a sphere contacting the fingerpad. Shown for an ideal population with uniform sensitivities (solid line and filled circles) and for three realistic populations (broken lines and open symbols). The characteristics of populations 1,2, and 3 are defined in Results. $A$, The $y$ component of the centroid plotted against the $y$ position of the stimulus. $B$, The second moment, which from Equation 4 has dimensions in $\mathrm{mm}^{-2}$, as a function of the curvature of the stimulus. Solid line with crosses shows that the $y$ component of the centroid is invariant with curvature. $C$, Weighted sum (dimensions in imp s $\mathrm{sm}^{-1}$ ).

there is a significant interaction between the curvature of the sphere and the sum, mainly because of the broad skirts in the response profiles for the less curved surfaces. The sum increases markedly with a decrease in curvature, whereas the published data of Goodwin and Wheat (1992) show only a minor interaction that is in the opposite direction. This problem is overcome by using a weighted sum of responses with a progressively decreasing contribution from afferents with receptive fields located at progressively increasing distances from the center of activity. An exponential weighting factor is used:

$$
w t s u m=\delta^{2} \sum_{i j} r_{\mathrm{ij}} e^{-0.667 d_{\mathrm{ij}}}
$$

where $d_{\mathrm{ij}}$ is the distance from the receptive field center of the fiber to the centroid of neural activity; thus, $d_{\mathrm{ij}}{ }^{2}=\left(x_{\mathrm{i}}-x_{\text {cent }}\right)^{2}+$ $\left(y_{\mathrm{j}}-y_{\text {cent }}\right)^{2}$.

There is no interaction between the centroid and curvature or force, nor between the second moment and position or force. Similarly, the weighted sum is not affected by position, but it is affected slightly by curvature for curvatures below $200 \mathrm{~m}^{-1}$.

Although the three measures illustrated in Figure 3 are simple, they reflect the three stimulus parameters that are different in nature. We use the measures to indicate the behavior of real neural codes for these parameters, allowing us to investigate the effects of the population characteristics on the neural representation of the parameters.

\section{Variation in fiber sensitivity}

There is a large variation in sensitivity among SAIs, and this results in "distortions" of the ideal population response. In two studies in the monkey, we found that SAI sensitivities $\left(s_{\mathrm{ij}}\right.$ in Eq. 2) were normally distributed, with a mean of 40 and an SD of 15.5 (Goodwin et al., 1995; Wheat et al., 1995), and data from humans are consistent with this (Goodwin et al., 1997). In our model, individual afferents are assigned sensitivities by a Gaussian random number generator, with a mean of 40 and an SD of 15.5. One set of sensitivities, generated in this way, are shown for fibers with receptive field centers along the $x$-axis (Fig. $4 A$ ), along the $y$-axis (Fig. $4 B$ ), and as a two-dimensional contour plot for the whole population (Fig. $4 C$ ). The population with the particular distri- bution of sensitivities shown in Figure $4, A-C$, is referred to as population 1 in the remainder of the paper. How do these variations in sensitivity affect the population response profiles? The response of each afferent in this population is given by the product of its sensitivity and the underlying Gaussian receptive field profile (Eq. 2). The broken lines in Figure 4, $D$ and $E$, show the Gaussian profile for a sphere of curvature $256 \mathrm{~m}^{-1}$, and the solid lines show the responses of the afferents to this sphere. For this population, the response profile is distorted and there is a lateral shift in the peak of activity, particularly in the $x$ direction (Fig. $4 F$ ).

A second set of sensitivities, with the same underlying distribution, defines population 2 in Figure 4, $G$ and $H$; this population exhibits a marked narrowing of the response profile, particularly in the $y$ direction. The third illustrative population (Fig. 4I) has a profile that exhibits less distortion than the previous two. Presumably, the spatial pattern of the distribution of sensitivities varies widely from finger to finger in humans; therefore, we generated a large number of patterns and selected these three examples for further analysis. Most patterns showed distortions of the order of that shown by population 3 , whereas populations 1 and 2 exhibit two of the more extreme cases of distortion.

The effects of these response profile distortions on our population measures are shown by the broken lines in Figure 3. It is obvious that, for each population, the measures reflect the stimulus parameters, despite the distortions in response profiles. In simple terms, although changes in sensitivity have a large effect on the responses of individual afferents, these effects tend to cancel when averaged over the whole population. Differences in vertical offsets for each population can easily be accounted for when scaling stimuli and, as will be shown later, do not affect the ability to discriminate stimuli.

\section{Response variability}

As presented so far, a population of SAIs has infinite resolution because arbitrarily small changes in a stimulus parameter will result in changes in the corresponding measure of the population response. In reality, resolution is limited by neural noise. Noise of some form occurs all the way along the pathway, starting with variations in skin mechanics and ending with noise in the final 


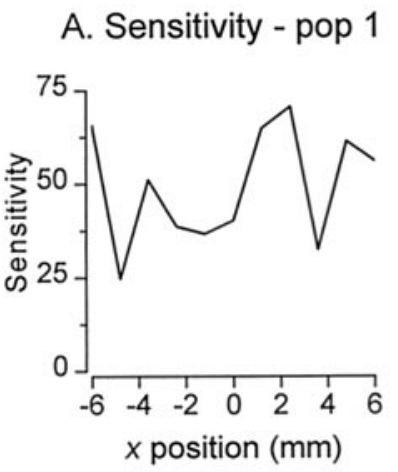

D. Response - pop 1

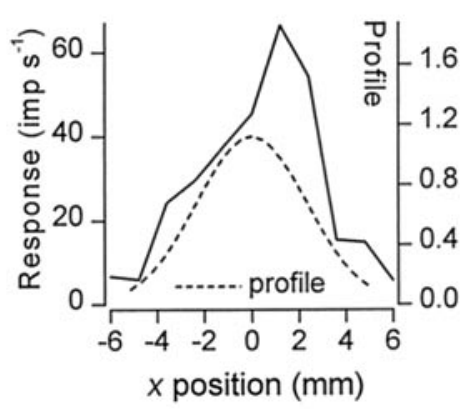

G. Response - pop 2

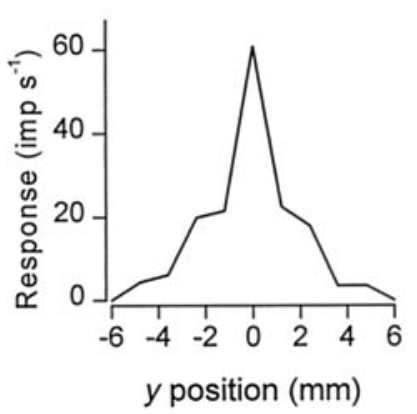

contours
B. Sensitivity - pop 1

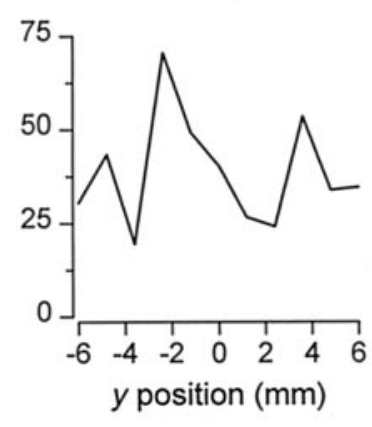

E. Response - pop 1

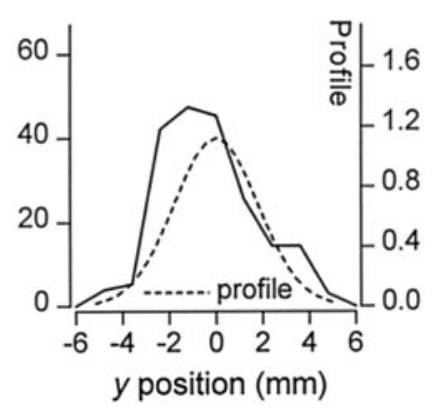

H. Response - pop 2

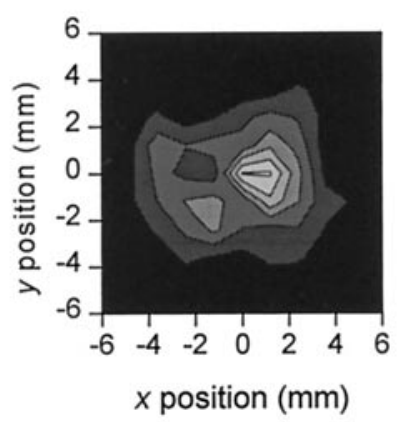

$20-30$

$30-40$

$40-50$

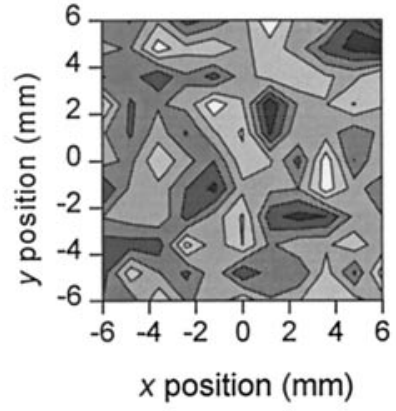

\section{Sensitivity - pop 3}

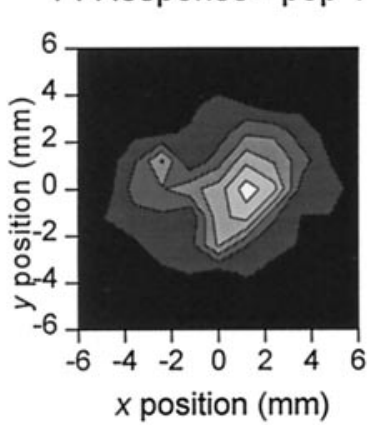

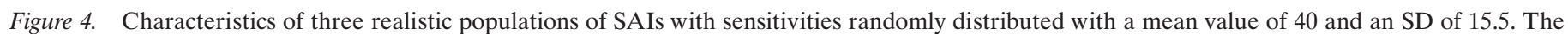

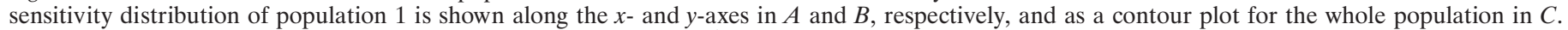

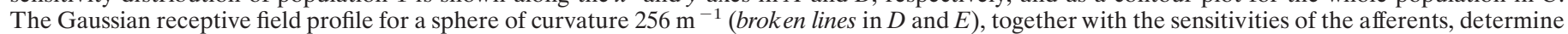

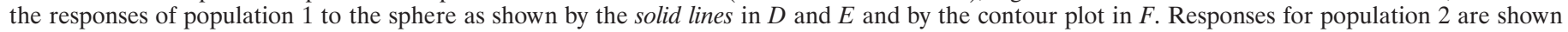
in $G$ and $H$. Sensitivities for population 3 are shown in $I$. The gray scale (for values) shown at the bottom is common to all contour plots.

decision process. Regardless of the source of the noise, it can be placed in one of two broad categories, namely, noise that is dependant on the level of neural activity and noise that is independent of the magnitude of the responses. These two categories are accounted for by modifying Equation 2 to:

$$
r_{\mathrm{ij}}=\left(1+\alpha_{\mathrm{ij}}\right) s_{\mathrm{ij}} k a e^{-\left(b x_{\mathrm{i}}^{2}+c y \mathrm{j}^{2}\right)}+\beta_{\mathrm{ij}}
$$

The first noise factor $\alpha_{\mathrm{ij}}$ is a normally distributed random variable, with a mean of 0 and an SD $\sigma_{\alpha}$ that is set as a parameter. The effect of this factor is that the response varies about its mean value such that the magnitude of the variation is proportional to the magnitude of the mean response (the coefficient of variation is $\sigma_{\alpha}$ regardless of the mean response). For convenience, we will refer to the factor $\alpha$ as "proportional noise." The second noise factor $\beta_{\mathrm{ij}}$ is a normally distributed random variable (independent of $\alpha$ ), with a mean of 0 and an $\operatorname{SD} \sigma_{\beta}$ (imp s${ }^{-1}$ ) set as a parameter. The effect of this noise factor is that the response of the afferent varies about its mean value such that the magnitude of the variation is independent of the magnitude of the mean response. For convenience, we will refer to the factor $\beta$ as "additive noise." In the initial analysis, the noise attributed to each afferent is independent of the noise on the other afferents; that is, the $\alpha_{\mathrm{ij}}$ are independent for all $i, j$ and similarly for the $\beta_{\mathrm{ij}}$. The peripheral component of noise has been estimated by experimental measurement as $\sigma_{\alpha}=0.03, \sigma_{\beta}=0$ (Wheat et al., 1995). Values of $\alpha$ and $\beta$ for the central components of noise are not known, so we illustrate a range of values that are consistent with published responses of somatosensory cortical neurons (see Discussion). 

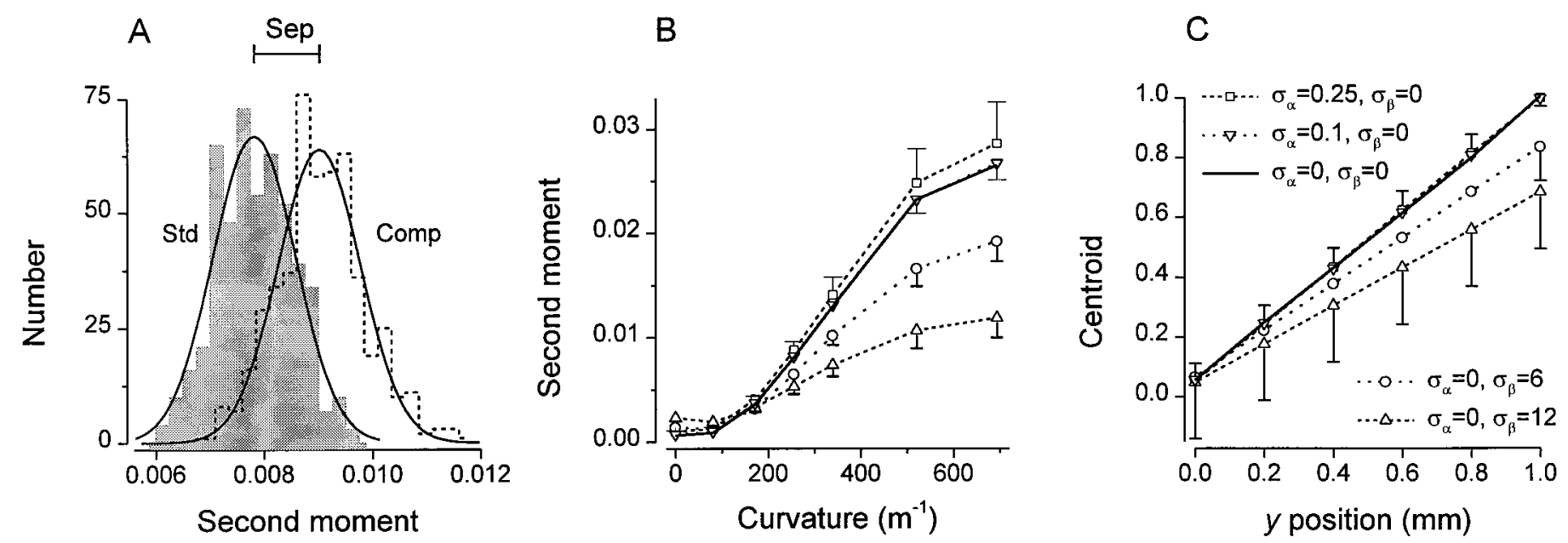

Figure 5. Effect of noise on measures of the population response. A, Distribution of second moments for 500 presentations of a standard sphere of curvature $287 \mathrm{~m}^{-1}$ (shaded histogram) and of a comparison sphere with a curvature, $316 \mathrm{~m}^{-1}$, that is $10 \%$ greater (open histogram). Solid lines are best-fit Gaussians. Noise level, $\sigma_{\beta}=6 \mathrm{imp} \mathrm{s}{ }^{-1}$. The decision boundary bnd is half the distance marked Sep. $B$, $C$, Second moment and centroid, respectively. Lines and data points show means, and error bars show unilateral SDs; $n=100$. For clarity, not all error bars are shown. For the solid line (no data points), there is no noise. Broken lines with down triangles and squares represent proportional noise with $\sigma_{\alpha}=0.1$ and 0.25 , respectively. Additive noise, independent of activity, with $\sigma_{\beta}=6$ and $12 \mathrm{imp} \mathrm{s}^{-1}$, are shown by broken lines with circles and up triangles, respectively. The centroid $(B)$ is shown for a sphere of curvature $172 \mathrm{~m}^{-1}$. For the population used in this simulation, all afferents had the same sensitivity (40).

In the presence of noise, repeated application of a single stimulus will result in a distribution of varying responses in each afferent and, therefore, any measure extracted from these responses will show a distribution of values as illustrated for the second moment in Figure $5 A$. In general, changing the noise level will affect both the mean and the SD of any measure calculated from the responses. For the second moment (Fig. 5B), activitydependent noise levels of $10 \%\left(\sigma_{\alpha}=0.1\right)$ hardly change the means of the measure, and the magnitudes of the SDs indicate the effect of the noise. Increasing the noise level to $25 \%$ shifts the means upward slightly and increases the SDs. In contrast, increasing the amount of noise that is independent of the level of responses $\left(\sigma_{\beta}=6\right.$ and $\left.12 \mathrm{imp} \mathrm{s}{ }^{-1}\right)$ results in a progressive compression of the function (reduction in signal), with little increase in the SDs. For the centroid (Fig. 5C), the overall characteristics are similar, but there are differences in detail. For example, increasing $\sigma_{\beta}$ from 6 to $12 \mathrm{imp} \mathrm{s}^{-1}$ increases the SD of the measure, and the compression of the function is less marked than it was for the second moment. For the weighted sum (data not shown), there is a negligible shift in means, and an increase in either type of noise increases the SD of the measure. Functions for the second moment and the centroid are compressed with additive noise because it tends to flatten the response profiles. In the model, afferents are not permitted to have negative responses; these are set to zero.

From the above illustrations, it is apparent that the effect of neural noise on each population response measure and its SD is complex and differs for the three stimulus parameters. To visualize the impact on tactile resolution, we have extracted from the model difference limens and Weber fractions analogous to those measured in our human psychophysics experiments. The principal is illustrated in Figure $5 A$ for curvature discrimination. The distributions of second moments for the standard and comparison stimuli are approximately normal and, as noise increases, the distributions increase in width. The discrimination threshold is calculated as described in Materials and Methods.

\section{Discrimination thresholds}

How is resolution affected by the two classes of noise, and what effect does the variation in sensitivity among afferents, which we have shown "distorts" the population responses, have on the discriminative capacities of specific populations? We computed the ability of the model to discriminate curvature using the same two standard spheres that we used in our psychophysics experiments (curvature 287 and $144 \mathrm{~m}^{-1}$ ). The results, expressed as a Weber fraction (difference limen divided by the value of the standard), should be viewed in light of the Weber fraction of $\sim 0.1$ measured by us in humans (Goodwin et al., 1991). For position discrimination, we computed the difference limens for spheres of curvature 172 and $521 \mathrm{~m}^{-1}$; the human difference limens we measured with these spheres were 0.55 and $0.38 \mathrm{~mm}$, respectively (Wheat et al., 1995). Weber fractions for the model were also computed for contact force, but we do not have directly comparable experimental measurements of human performance. The nearest equivalent is the Weber fraction of 0.134 reported by Brodie and Ross (1984) for a 50 gm weight placed on the subject's palm.

The resolution of population 3 (Fig. 4I), which exemplifies a distribution of sensitivities that results in a typical degree of distortion of the response profiles, is shown in Figure 6 for combinations of the two types of noise. Resolution decreases with an increase in either type of noise for all three stimulus parameters, but there are differences in effect because of the different natures of the corresponding stimulus measures. For position discrimination, the effect of proportional noise decreases with increasing additive noise, whereas for curvature discrimination, the effects of the two types of noise are approximately additive over the whole range. For force discrimination, proportional noise with an SD of 0.5 swamps the effect of additive noise.

It is obvious from Figure 6 that, with peripheral noise alone $\left(\sigma_{\alpha}\right.$ $\left.=0.03, \sigma_{\beta}=0\right)$, resolution far exceeds the resolution of human subjects. Thus, the model shows clearly that the limiting factor for humans is central noise of some form. The model also indicates 


\section{A. Curvature}

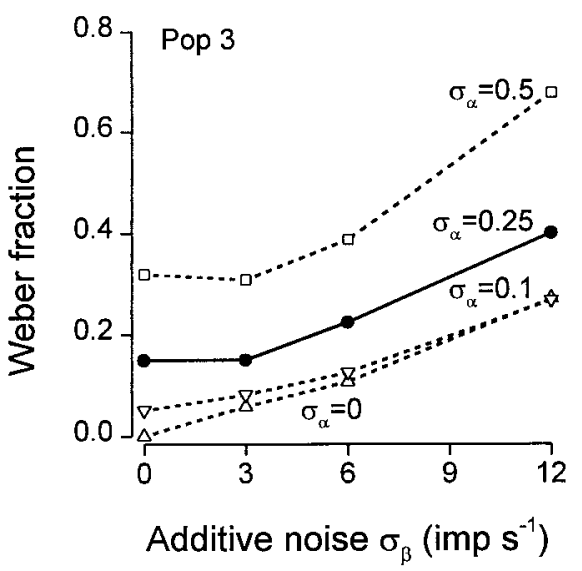

B. Position

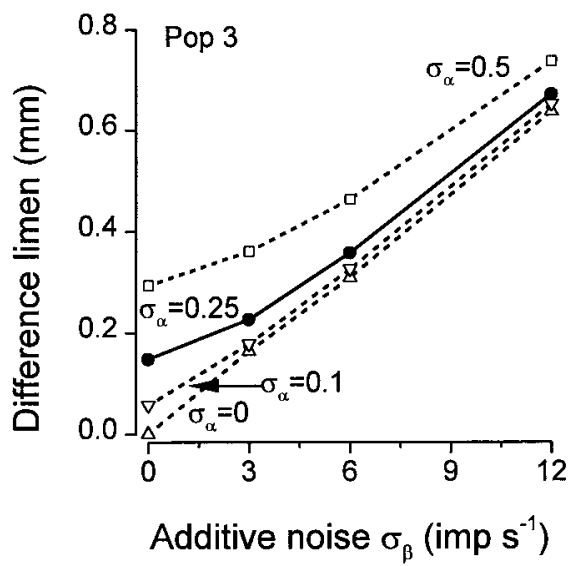

C. Force

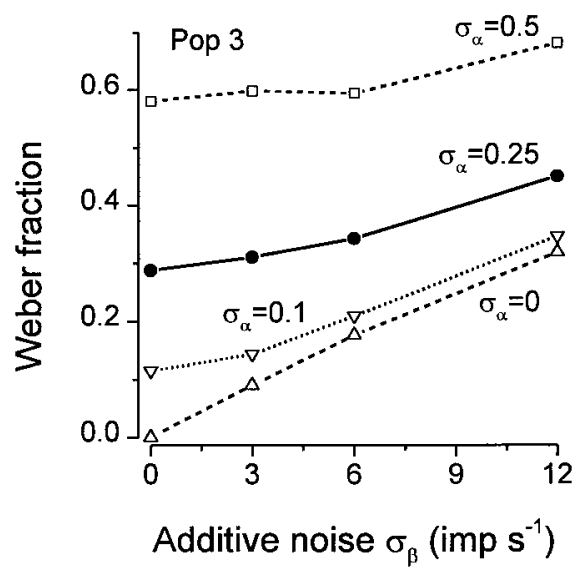

Figure 6. Discrimination performance for the representative population 3. The extent of additive and proportional noise is specified by their SDs $\sigma_{\beta}$ and $\sigma_{\alpha}$, respectively. $A$, Weber fraction for curvature using a standard curvature of $287 \mathrm{~m}^{-1}$. $B$, Difference limen for the position of a sphere of curvature $172 \mathrm{~m}^{-1} . C$, Weber fraction for the contact force of a sphere of curvature $256 \mathrm{~m}^{-1}$; the standard contact force is $10 \mathrm{gf}$.

A. Curvature discrimination

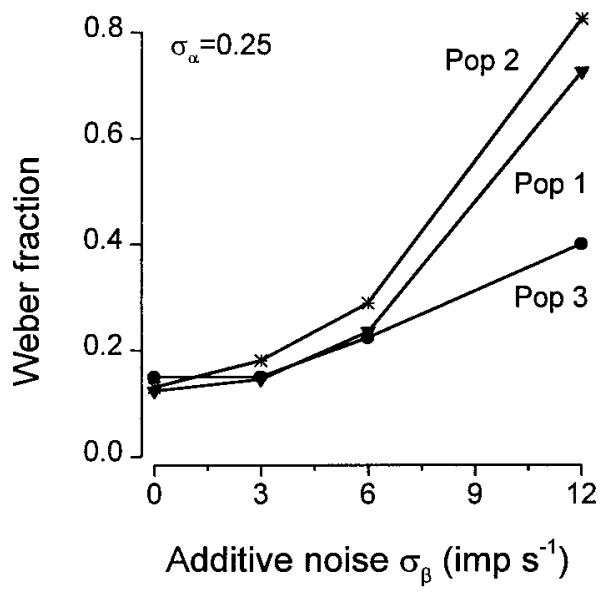

B. Position discrimination

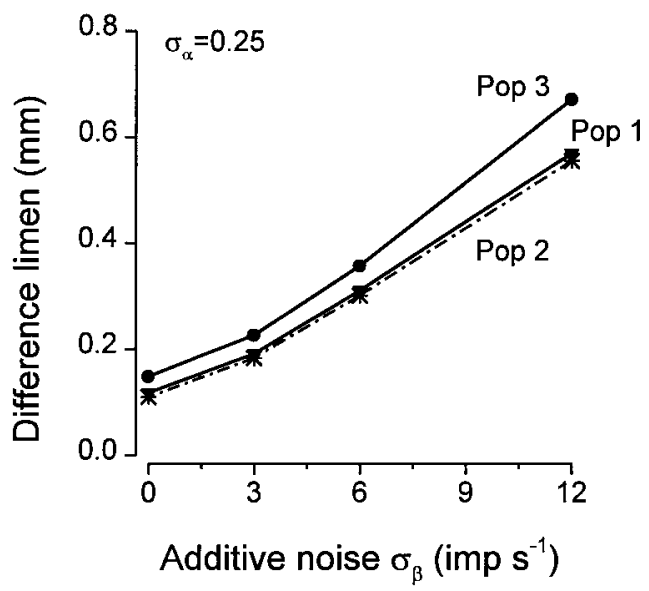

Figure 7. Discrimination performance compared for population 3, which produces an average degree of response profile distortion, and populations 1 and 2, which result in more extreme distortions. Proportional noise is set at $\sigma_{\alpha}=0.25 . A$, Weber fraction for curvature discrimination with a standard curvature of $287 \mathrm{~m}^{-1} . B$, Difference limen for the position of a sphere of curvature $172 \mathrm{~m}^{-1}$.

that the two forms of noise affect different measures differently. For example, to match the human Weber fraction for curvature of $\sim 10 \%$, the SDs for additive noise and proportional noise must be less than $6 \mathrm{imp} \mathrm{s}^{-1}$ and 0.25 , respectively, whereas the human difference limen for position, $0.55 \mathrm{~mm}$, is easily achieved if additive noise has an SD less than $6 \mathrm{imp} \mathrm{s}{ }^{-1}$, even in the presence of high levels of proportional noise.

It is possible that, in some human fingerpads, the SAI population may have a sensitivity distribution of an extreme nature, resulting in a greater than average distortion of the response profiles. The model indicates the consequences of this as seen by comparing the resolution of population 3 with the resolution of populations 1 and 2 (which have greater distortion) in Figure 7. Position discrimination is not affected much by the population sensitivity characteristics, and, in fact, the resolution is fortuitously slightly better for populations 1 and 2, despite their greater distortion compared with population 3 (Fig. 4). Curvature discrimination is also not affected much, except at high levels of additive noise in which case populations 1 and 2 show a decreased resolution.

In our human psychophysics experiments, discrimination of curvature and of position were measured for two different spheres. The Weber fraction for curvature was $\sim 10 \%$ for both standards used, 287 and $144 \mathrm{~m}^{-1}$. With a small amount of proportional noise $\left(\sigma_{\alpha}=0.1\right)$, the Weber fractions produced by the model for curvature discrimination (Fig. $8 A$ ) do not depend on the curvature of the standard, although with more proportional noise $\left(\sigma_{\alpha}=0.25\right)$, performance is slightly better for the less curved sphere. Thus, human performance and performance of the model are similar.

For position discrimination, the difference limen produced by the model (Fig. $8 B$ ) is smaller for the less curved sphere. This is the reverse of our finding in human psychophysics experiments in which the difference limen was lower for the more curved sphere. Thus, our subjects could not have used a code with the characteristics of the centroid. Instead, we suggest that the subjects used 


\section{A. Second moment}

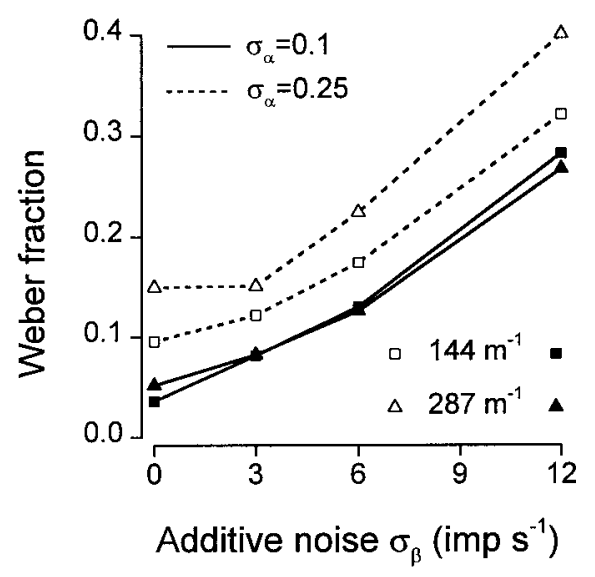

B. Centroid

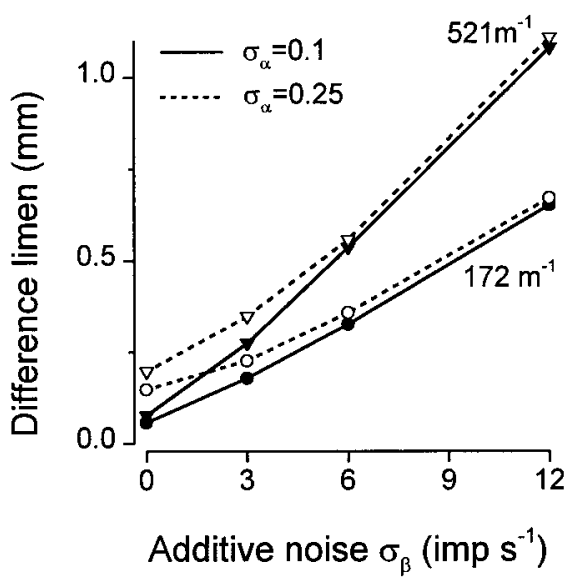

C. Volume

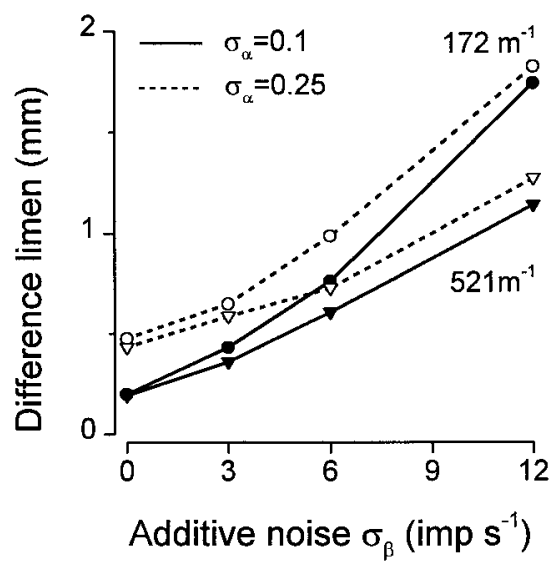

Figure 8. Discrimination of the curvature and position of a sphere by population 3. Proportional noise SD was 0.1 (solid lines) or 0.25 (broken lines), and additive noise SD ranged from 0 to 12 . $A$, Weber fraction for curvature based on the second moment of the response. Two standards were used with

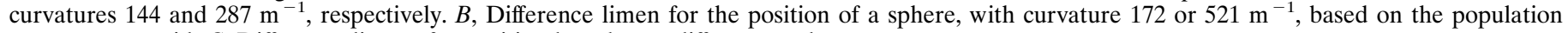
response centroid. $C$, Difference limens for position based on a difference volume measure.

a different, nonspecific cue that exists in a two-alternative forcedchoice design for changes in position like that used in our experiments. If the standard and comparison stimuli are presented at different positions on the finger, then the shift in the population response can be exploited without determining the absolute positions of the two stimuli. As a surrogate code for this type of strategy, we have used the volume between the response to the standard and the response to the comparison stimulus.

The difference volume can be calculated from the model as:

$$
v o l=\delta^{2} \sum_{i j}\left|r_{\mathrm{ij}}-R_{\mathrm{ij}}\right|
$$

where $r_{\mathrm{ij}}$ and $R_{\mathrm{ij}}$ are the responses of the fiber with a receptive field center at position $\left(x_{\mathrm{i}}, y_{\mathrm{j}}\right)$ to the first and second stimulus, respectively, in the pair being compared. Difference limens for position computed from this volume measure are shown in Figure $8 C$; performance of the model is better for the more curved sphere as was the case for human performance, suggesting that a strategy of this nature was used by our subjects. Moreover, the magnitudes of these difference limens are close to those of the human for noise levels up to $\sigma_{\alpha}=0.25$ and $\sigma_{\beta}=6$. Codes bases on measures such as the difference volume are not useful in everyday multi-dimensional tasks because such a difference volume will result from a change in any stimulus parameter or combination of stimulus parameters. However, they can be used effectively in a forced-choice paradigm in which only one stimulus parameter is varied. The results illustrated for population 3 in Figure 8 held for the other populations as well.

\section{Response covariance}

In all computations performed so far, we have assumed that the noise contributed by each afferent is independent of that contributed by the other afferents, and thus the variables $\alpha_{\mathrm{ij}}$ for all $i, j$ were independent of each other as were the variables $\beta_{\mathrm{ij}}$. However, such independence is unlikely, and correlation among the variation of responses of the afferents has a significant effect on the resolution of the population. This type of correlation is not to be confused with the fact that a change in a stimulus parameter will produce correlated changes in the responses of the afferents by virtue of Equation 2; for example, a decrease in $k$ will reduce the response in all afferents. Here, we are concerned with correlation among the random variables $\alpha_{\mathrm{ij}}$ and among the random variables $\beta_{\mathrm{ij}}$, which does not depend on the stimulus parameters. There have been a number of theoretical studies of the effect of such covariance but only for codes, or population measures, that are effectively sums of the responses of all the afferents and usually for conditions in which each of the afferents has a similar response. In such cases, the resolution of the population decreases as the covariance increases (Johnson et al., 1979; Gawne and Richmond, 1993; Zohary et al., 1994; Shadlen et al., 1996). In contrast, Johnson (1980b) has pointed out that, for spatial population codes, covariance will improve the resolution, but there have been no detailed analyses.

The effect of correlation on resolution is not amenable to experimental analysis, but the model provides a way of investigating this issue. In this section, the random variables are constructed in such a way that the correlation coefficients for pairs of $\alpha_{\mathrm{ij}}$ (for all $i$ and $j$ ) are $0,0.2$, or 0.8 , and similarly for $\beta_{\mathrm{ij}}$. Three representative levels of noise are illustrated: additive noise alone $\left(\sigma_{\alpha}=0, \sigma_{\beta}=6\right)$, proportional noise alone $\left(\sigma_{\alpha}=0.25, \sigma_{\beta}=0\right)$, and a combination of additive and proportional noise $\left(\sigma_{\alpha}=0.25\right.$, $\left.\sigma_{\beta}=6\right)$.

The measure for contact force, a weighted sum, has many of the features of a total population response, although a weighting factor is present and, unlike the usual theoretical treatments, all afferents do not have the same mean response. Thus, an increase in covariance results in a decrease in the resolution of force (an increase in the Weber fraction) for both types of noise (Fig. 9C). The population measure used to indicate position is a spatial code; therefore, resolution increases as the covariance increases for both types of noise (Fig. 9B). The second moment, used as a measure for curvature, is a more complex code with both spatial components and components that depend on overall responses; it is not easy to predict from Equation 4 how covariance will affect resolution. This measure is affected differently by the two types of noise (Fig. 9A). For proportional noise, the spatial effects dominate, and increasing covariance improves resolution. For additive 
A. Curvature

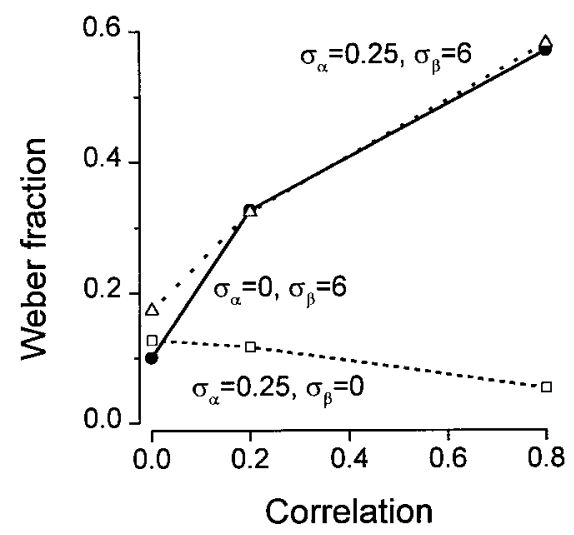

B. Position

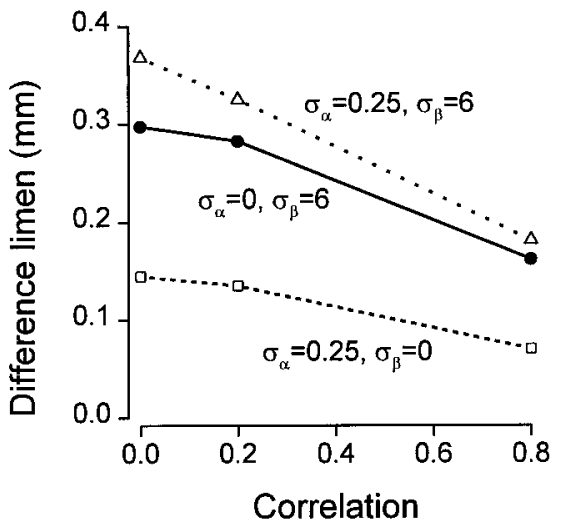

C. Force

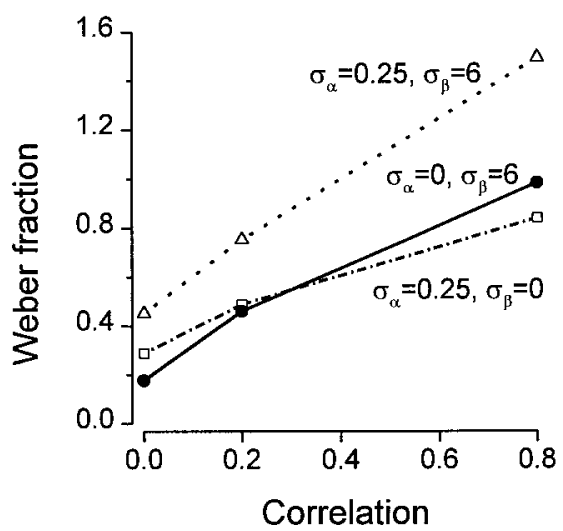

Figure 9. Effect of covariance on the resolution of the second moment $(A)$, the centroid $(B)$, and the weighted sum $(C)$ of the population response. Noise was additive with an SD of 6, proportional with an SD of 0.25 , or a combination of the two. The random variables $\alpha_{\mathrm{ij}}$ were constructed such that the correlation coefficient between pairs of $\alpha_{\mathrm{ij}}$ was $0,0.2$, or 0.8 for all $i, j$ pairs, and similarly for $\beta_{\mathrm{ij}} . A$, Standard curvature $287 \mathrm{~m}^{-1} . B$, Curvature 172 $\mathrm{m}^{-1}$. C, Curvature $256 \mathrm{~m}^{-1}$, standard force $10 \mathrm{gf}$.

noise, the overall responses dominate and the resolution decreases. A major reason for this is that previously inactive afferents around the skirts of the population response become active and are recruited by additive noise. When both types of noise are present and covariance is nonzero, the effects of additive noise swamp the effects of proportional noise on the second moment.

Because the level of noise is so low in the periphery $\left(\sigma_{\alpha}=\right.$ 0.03 ), these effects of correlation must be occurring centrally.

\section{Innervation density}

The innervation density of the afferent fibers will affect the resolution of the system, but in a way that is not obvious. The effect of changes in spatial sampling on the behavior of spatial codes is analyzed here using the measure for the position of the stimulus. Human discrimination of position is "hyperacute" (Wheat et al., 1995). For nonspatial codes, represented here by the measure for contact force, the effect of innervation density is mediated through a change in statistical reliability resulting from a change in the number of contributing fibers. More complex codes, represented here by the measure for curvature, will be affected by both spatial sampling changes and changes in the number of fibers.

So far, the SAI populations have been reconstructed with the default innervation density of $0.7 \mathrm{~mm}^{-2}$, which is the value estimated by Johansson and Vallbo (1979) for the human fingertip. Assuming a uniform square arrangement of receptive field centers, this corresponds to a spacing of $1.2 \mathrm{~mm}$ between adjacent centers. To examine the effect of innervation density, we increased it by $84 \%$ to $1.29 \mathrm{~mm}^{-2}$ (spacing $0.88 \mathrm{~mm}$ ) and decreased it to 66 and $20 \%$ of the default value, $0.465 \mathrm{~mm}^{-2}$ (spacing 1.47 $\mathrm{mm}$ ) and $0.143 \mathrm{~mm}^{-2}$ (spacing $2.64 \mathrm{~mm}$ ), respectively. In each case, the area of skin was kept constant at $13.2 \times 13.2 \mathrm{~mm}$ so that the potential total number of fibers was 121 for the default density of $0.7 \mathrm{~mm}^{-2}$ and was 225,81 , and 25 for the densities of 1.29 , 0.465 , and $0.143 \mathrm{~mm}^{-2}$, respectively. Two important aspects of the size of the population, not usually accounted for, should be stressed. First, the size is an upper bound, but the actual size will often be smaller because not all fibers will be active; moreover, the size will vary from trial to trial because of the effects of random noise. This is true in both the model and real life. Second, the number of afferents is relatively small.

When the noise associated with the afferents is uncorrelated, behavior of the weighted sum is consistent with predictions based on the size of the population (Fig. 10C). Resolution improves with an increase in innervation density (decrease in spacing) when the noise is additive and even more so for proportional noise. For the centroid, the situation is more complex (Fig. 10B). For additive noise, increasing innervation density increases resolution, but for proportional noise, the effect is smaller and inconsistent. Local increases and decreases in resolution reflect the characteristics of specific populations in that the variations in sensitivity of the afferents may lead to spurious increases in resolution, with decreases in density such as that seen in Figure $10 B$ when spacing increases from 1.2 to $1.47 \mathrm{~mm}$. In all cases, position resolution greatly exceeds that predicted by simplistic application of the sampling theorem (i.e., twice the afferent spacing). For the second moment, changing innervation density to approximately half or double the default value has small and inconsistent effects, again depending on the characteristics of the particular population (Fig. 10 $\mathrm{A}$ ).

With an increase in the correlation of the noise, there is a fundamental difference in the behavior of measures, which are analogous to the summed activity of the afferents and the behavior of measures based on the spatial characteristics of the population. Resolution for contact force decreases as covariance increases, and the effect of changing innervation density diminishes (Fig. 10C,F,I). This is consistent with previous studies, which have shown that, for total response codes, resolution improves with the number of fibers in the population when noise is uncorrelated but diminishes and becomes independent of the population size as correlation increases (Zohary et al., 1994). The behavior of the centroid, which is a spatial code, is quite different. As covariance increases, resolution for the position of the stimulus increases, and at all values of the correlation coefficient, position resolution increases with increasing innervation density (Fig. 10B,E,H). The measure for the curvature of the stimulus, the second moment, has attributes of both spatial codes and total 
Figure 10. Effect of innervation density on resolution. The four densities tested, $1.29,0.70,0.465$, and $0.143 \mathrm{~mm}^{-2}$, correspond to a spacing between adjacent receptive field centers of $0.88,1.2,1.47$, and $2.64 \mathrm{~mm}$, respectively. Resolution of the second moment $(A, D, G)$ is shown by the Weber fraction for a curvature of $287 \mathrm{~m}^{-1}$. Resolution of the centroid $(B$, $E, H)$ is shown by the difference limen for the position of a sphere of curvature $172 \mathrm{~m}^{-1}$. The Weber fraction for a contact force of $10 \mathrm{gf}$ shows the resolution of the weighted sum $(C, F, I)$. Two types of noise were used: additive noise $\left(\sigma_{\beta}=\right.$ $\left.6 \mathrm{imp} \mathrm{s}^{-1}\right)$ and proportional noise $\left(\sigma_{\alpha}=\right.$ $0.25)$. The correlation coefficient $(r)$ between the noise at each pair of fibers was $0,0.2$, or 0.8 . In $I$, the spurious decrease in Weber fraction with an increase in spacing (solid line) is a peculiarity of the particular populations; on average, this curve would tend to a horizontal line.
A. Curvature, $r=0$

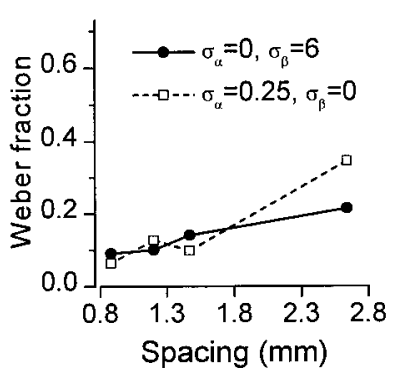

D. Curvature, $r=0.2$

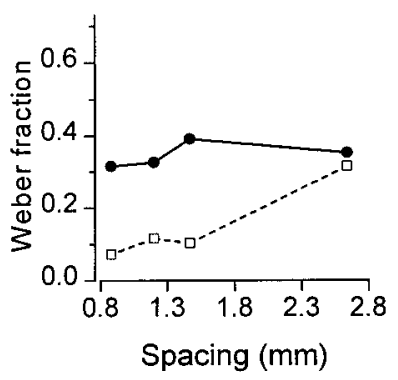

G. Curvature, $r=0.8$

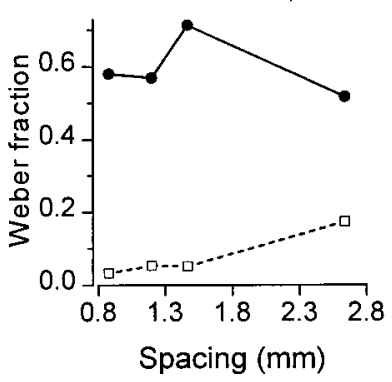

B. Position, $r=0$

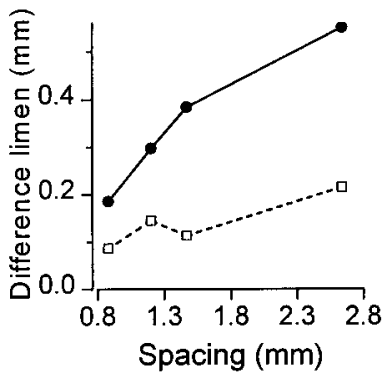

E. Position, $r=0.2$

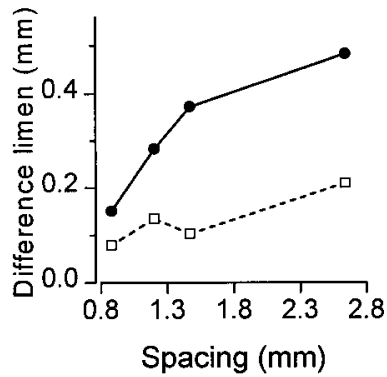

H. Position, $r=0.8$

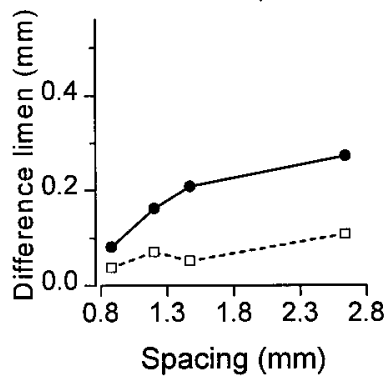

C. Force, $r=0$

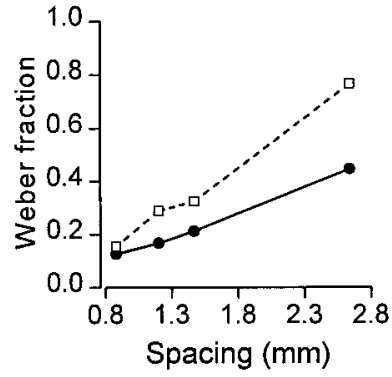

F. Force, $r=0.2$

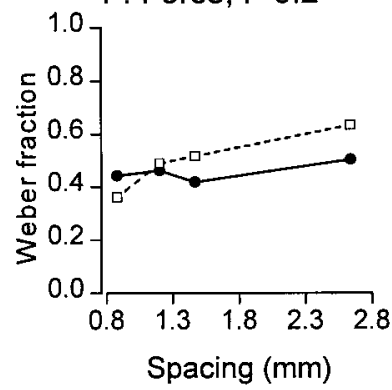

I. Force, $r=0.8$

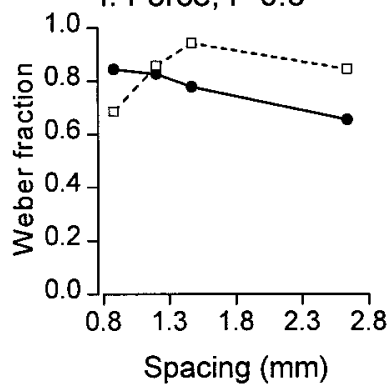

response codes, and therefore its behavior is a mixture of the behaviors of the codes for force and position (Fig. 10A,D, G).

The model shows clearly, as seen in Figure 10, that the effects of innervation density, which is a peripherally determined population parameter, are determined primarily by the characteristics of noise, which is of central origin.

\section{Innervation geometry}

Details of the geometric arrangement of SAI receptive field centers in the human fingerpad are not known, but it is unlikely to be precisely uniform. Also, it is known that, with aging or injury, some receptors or fibers are lost, presumably in a nonuniform manner. Thus, it is important to know how variations in the innervation geometry affect the resolution of the population.

Positions of receptive field centers in population 3 were randomized by adding a normally distributed random variable to the $y$ position of each receptive field center (Fig. 11A). Positions were perturbed in the $y$ direction because, in both our human psychophysics experiments and in this simulation, the position of the sphere was varied in the $y$ direction. The overall innervation density is still $0.7 \mathrm{~mm}^{-2}$, but there is considerable variation in local density. Receptive field centers (Fig. 2) still lie along columns in the matrix with constant $x$ values $\left(x_{\mathrm{i}}\right)$ but do not lie in rows with constant $y$ values $\left(y_{\mathrm{j}}\right)$; instead, each $y$ value is different and is therefore denoted $y_{\mathrm{ij}}$. For nonuniform spacing, a slight elaboration of Equations 3-5 is needed to calculate true estimates of the centroid, second moment, and weighted sum as follows.

$$
\begin{gathered}
\sum_{i} y_{\mathrm{i} 1} r_{\mathrm{i} 1}\left(y_{\mathrm{i} 2}-y_{\mathrm{i} 1}\right)+0.5 \sum_{i} \sum_{j=2}^{k-1} y_{\mathrm{ij}} r_{\mathrm{ij}} \\
y_{\mathrm{cent}}=\frac{\left(y_{\mathrm{i}, \mathrm{j}+1}-y_{\mathrm{i}, \mathrm{j}-1}\right)+\sum_{i} y_{\mathrm{ik}} r_{\mathrm{ik}}\left(y_{\mathrm{ik}}-y_{\mathrm{i}, \mathrm{k}-1}\right)}{\sum_{i} r_{\mathrm{i} 1}\left(y_{\mathrm{i} 2}-y_{\mathrm{i} 1}\right)+0.5 \sum_{i} \sum_{j=2}^{k-1} r_{\mathrm{ij}}} \\
\left(y_{\mathrm{i}, \mathrm{j}+1}-y_{\mathrm{i}, \mathrm{j}-1}\right)+\sum_{i} r_{\mathrm{ik}}\left(y_{\mathrm{ik}}-y_{\mathrm{i}, \mathrm{k}-1}\right) \\
\text { secmom }=\frac{\left(y_{\mathrm{i}, \mathrm{j}+1}-y_{\mathrm{i}, \mathrm{j}-1}\right)+\sum_{i}\left(r_{\mathrm{ik}}-\bar{r}\right)^{2}\left(y_{\mathrm{ik}}-y_{\mathrm{i}, \mathrm{k}-1}\right)}{\sum_{i}\left(r_{\mathrm{i} 1}-\bar{r}\right)^{2}\left(y_{\mathrm{i} 2}-y_{\mathrm{i} 1}\right)+0.5 \sum_{i} \sum_{j=2}^{k-1}\left(r_{\mathrm{ij}}-\bar{r}\right)^{2}} \\
\delta_{\mathrm{x}}\left[\sum_{i} r_{\mathrm{i} 1}\left(y_{\mathrm{i} 2}-y_{\mathrm{i} 1}\right)+0.5 \sum_{i} \sum_{j=2}^{k-1} r_{\mathrm{ij}}\right. \\
\left.\left(y_{\mathrm{i}, \mathrm{j}+1}-y_{\mathrm{i}, \mathrm{j}-1}\right)+\sum_{i} r_{\mathrm{ik}}\left(y_{\mathrm{ik}}-y_{\mathrm{i}, \mathrm{k}-1}\right)\right]^{2} \\
\text { wtsum }_{\delta_{\mathrm{x}}} \sum_{i} r_{\mathrm{i} 1}\left(y_{\mathrm{i} 2}-y_{\mathrm{i} 1}\right) e^{-0.667 d_{\mathrm{i} 1}}+0.5 \delta_{\mathrm{x}} \sum_{i} \sum_{j=2}^{k-1} r_{\mathrm{ij}} \\
\left(y_{\mathrm{i}, \mathrm{j}+1}-y_{\mathrm{i}, \mathrm{j}-1}\right) e^{-0.667 d_{\mathrm{ij}}}+\delta_{\mathrm{x}} \sum_{i} r_{\mathrm{ik}}\left(y_{\mathrm{ik}}-y_{\mathrm{i}, \mathrm{k}-1}\right) e^{-0.667 d_{\mathrm{ik}}}
\end{gathered}
$$




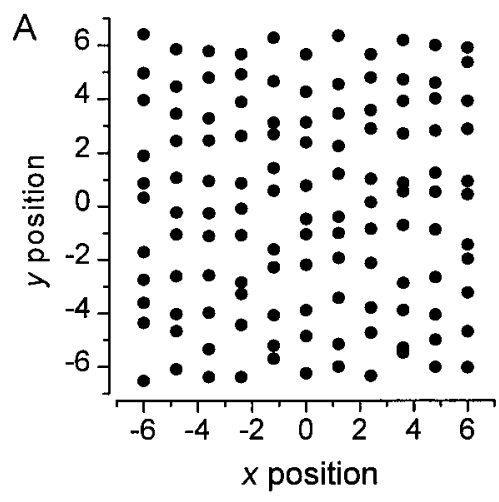

C. Curvature

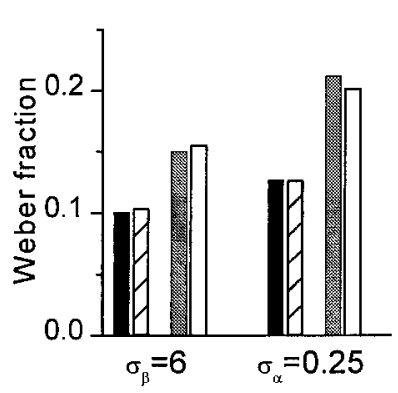

D. Position

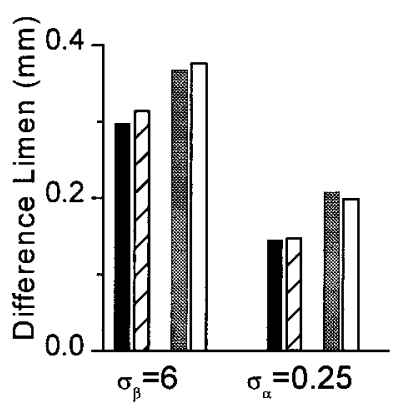

E. Force

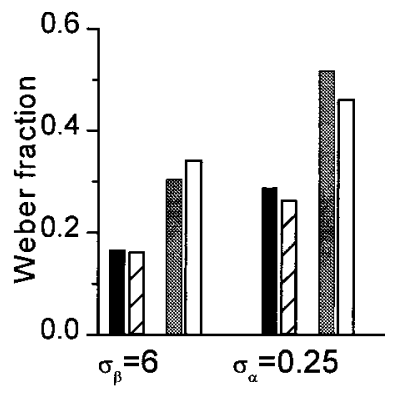

$1.2 \mathrm{~mm}$ uniform

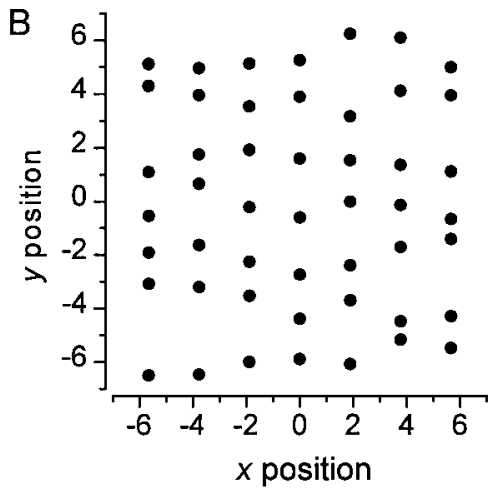

$1.2 \mathrm{~mm}$ scattered

20.2. $1.89 \mathrm{~mm}$ uniform

$1.89 \mathrm{~mm}$ scattered

Figure 11. Resolution in populations with nonuniform patterns of innervation. $A$, Positions of receptive field centers in population 3 were modified by randomizing their $y$ positions. Overall innervation density is $0.7 \mathrm{~mm}^{-2}$, corresponding to an average spacing between centers of $1.2 \mathrm{~mm}$. $B$, The overall innervation density of this population is $0.281 \mathrm{~mm}^{-2}$ (average spacing $1.89 \mathrm{~mm}$ ). $C-E$, Resolution for curvature, position, and contact force measured by the second moment, centroid, and weighted sum, respectively. For an innervation density of $0.7 \mathrm{~mm}^{-2}$, resolution for the population in $A$ (striped bars) is compared with resolution for the same population spaced uniformly ( filled black bars). The open bars and gray bars, respectively, compare resolution for the population in $B$ and resolution for the same population with uniform spacing (density $\left.0.281 \mathrm{~mm}^{-2}\right)$. Both additive noise $\left(\sigma_{\alpha}=0, \sigma_{\beta}=6\right)$ and proportional noise $\left(\sigma_{\alpha}=0.25, \sigma_{\beta}=0\right)$ are shown.

The effect that this scattering of receptive fields has on resolution can be seen by comparing the filled black bars and the striped bars in Figure 11, $C-E$. For both additive and proportional noise, with zero covariance between the afferents, nonuniformity in the receptive field geometry has a negligible affect on the resolution of curvature, position, or force. This is also true at a lower innervation density $\left(0.281 \mathrm{~mm}^{-2}\right)$, as shown by the gray bars and open bars in Figure 11. The same analysis was done with the noise correlated among afferents, and the results were equivalent to those in Figure 11. Regardless of the nature of the neural noise, resolution was hardly affected by a nonuniform pattern of innervation.

\section{DISCUSSION}

Previous studies of the responses of SAIs to spatially complex stimuli have shown that the various features of the stimulus are represented in the whole population response (LaMotte and Srinivasan, 1987b, 1996; Ray and Doetsch, 1990; Phillips et al., 1992; Cohen and Vierck, 1993; Blake et al., 1997; Dodson et al., 1998; Khalsa et al., 1998). However, these studies did not quantify the effects of inherent population characteristics, such as the pattern and density of innervation, on the representation or encoding of stimulus parameters. We tackled this problem directly by simulating the responses of the SAI population to a sphere contacting the fingerpad and by comparing neural measures with human performance for the perception of the curvature of the sphere, its position on the skin, and the contact force.

\section{Value of the model}

Quantitative comparison of neural and psychophysical data, which allows rigorous hypothesis testing, is essential for gaining insight into the behavior of the tactile system. Factors, such as innervation density, which have a profound effect on the system but are not seen in single-unit responses, must be accounted for.

A modeling approach permits an analysis of the consequences of the population characteristics, and its power and value are illustrated by a few examples from Results. (1) When experimentally measured values of innervation density and peripheral fiber noise are accounted for, the resolution of the population of SAIs far exceeds human performance (Fig. 6), demonstrating that the limiting factors occur in the central processing. (2) Variations in the pattern of innervation of the skin do not affect resolution (Fig. 11). (3) When subjects are highly trained in forced-choice paradigms, they may use singular cues to achieve levels of performance superior to those predicted from neural measures applicable to more general tasks (Fig. 8). 


\section{Neural measures}

We proposed three neural measures that we used as surrogate codes for the real neural measures underlying human perception of the three stimulus parameters. The centroid of the population response corresponds to the position of the sphere on the skin, the second moment of the response about its mean reflects the curvature of the stimulus, and the weighted sum of responses parallels the contact force. We do not imply that these are the actual codes used by the brain; rather, they are measures that behave in a similar manner to the real codes and that therefore allow us to contrast the effects of the population characteristics on the three types of codes that are different in nature. Testing specific candidate codes will require more systematic studies.

The sensitivity of SAIs in human fingerpads varies widely (Knibestol, 1975; Goodwin et al., 1997) so that profiles of response across the population are distorted (Fig. 4). Moreover, the distortion will be different for different fingers. Despite this marring of the peripheral neural representation, humans are able to discriminate stimuli with high fidelity. The three measures used by us are robust and insensitive to the inhomogeneity in afferent sensitivity. Simplistic neural codes, which are viable in ideal populations, cannot be used for real human fingers. For example, the profile distortions preclude using the location of the receptive field center of the most active fiber to determine the position of a sphere on the skin.

In principle, it is possible that the variation in sensitivity of the afferents is compensated for by complementary strengths at synapses in the CNS. In such a case, the effective population response profiles would correspond to those of a population with uniform sensitivity. Because neural measures, such as those proposed here, are effective despite sensitivity variations, the gain in resolution from such a strategy would not be great.

\section{Response variability}

The reliability with which information about a stimulus is relayed is limited by the inherent variability in responses resulting from factors such as variation in skin mechanics, noise in membrane potentials, and synaptic noise. At the primary afferent level of the somatosensory system, noise levels are low. We have measured a value of $\sigma_{\alpha}=0.03, \sigma_{\beta}=0$ for our stimuli (Wheat et al., 1995), which is consistent with the data of Edin et al. (1995) for brushes moving over the skin and the results of Johnson et al. (1979) for thermal stimuli. We have been unable to locate detailed and specific central noise measurements for any somatosensory stimuli. However, inspection of published trial-by-trial raster plots for somatosensory cortical cells (Gardner and Costanzo, 1980; Tremblay et al., 1996) shows noise values consistent with the ranges used in the model. For example, in Figure 2 of Whitsel et al. (1978), the response to a stimulus repeated 25 times had a mean \pm SD of $39.1 \pm 8.8$ impulses. The detailed variability analysis of Whitsel et al. (1977) and Schreiner et al. (1978) does not address the variability of responses to repeated stimuli, but rather the "variability" or distribution of the interspike intervals for a single stimulus.

In the visual cortex and motor cortex, it has been shown that variability of response increases as the response of the neuron increases (Heggelund and Albus, 1978; Dean, 1981; Vogels et al., 1989; Snowden et al., 1992; Lee et al., 1998). In general, the mean number of spikes and its variance are proportional. For retinal ganglion cells and neurons in the lateral geniculate nucleus, the extent of variability is independent of the magnitude of the response (Schiller et al., 1976; Croner et al., 1993; Edwards et al., 1995).

Together, the above data suggest that our partitioning of noise into proportional and additive components is an appropriate starting point and that the ranges of noise illustrated are reasonable. Other forms of noise, such as response variance proportional to response magnitude, may also occur. The lack of precise information on somatosensory noise weakens the model to some degree. There is a need for such experimental measurements that would enhance the power of the model and improve it by allowing subdivision of the lumped noise into components corresponding to the various levels in the CNS.

\section{Correlation}

Correlation of noise among central neurons has only been estimated in a few specific situations for a relatively small number of neurons; the reported correlations vary widely (Gawne and Richmond, 1993; Zohary et al., 1994; Lee et al., 1998). Because the details of correlation coefficients in the somatosensory system are not known, we have illustrated a full range from 0 through 0.2 up to 0.8 . Our aim is to delineate what effects increasing correlation has on the three types of measures rather than to imply that particular values exist. Correlation among lumped noise is a simplification of the real situation, but it serves to highlight the major effects of correlation on different types of neural measures. An increase in correlation decreased discrimination capacities for the weighted sum, in keeping with theoretical analyses and simulations for other neural codes based on sums or averages across the population (Johnson et al., 1979; Gawne and Richmond, 1993; Zohary et al., 1994; Shadlen et al., 1996). However, the reverse was true for the centroid, which is a neural measure relying on spatial patterns within the population; here, an increase in correlation among noise increased resolution. Abbott and Dayan (1999) have also shown that correlation can improve the accuracy of a population code.

Because noise levels are low in the periphery, these effects of correlation would be occurring in the CNS, and the model highlights the need for experimental measurements of covariance among somatosensory cortical neurons.

\section{Innervation density}

Johansson and Vallbo (1979) estimated the density of SAIs from the fingertip in humans as $0.7 \mathrm{~mm}^{-2}$, and Darian-Smith and Kenins (1980) estimated a similar density in the monkey. Assuming that receptive field centers lie in a uniform square matrix, the sampling theorem limits spatial resolution in general to 0.417 cycles/mm or to a period of $2.4 \mathrm{~mm}$. Specific tasks, such as position discrimination, are limited by other factors. The human difference limen of $\sim 0.5 \mathrm{~mm}$, so called hyperacuity (Loomis and Collins, 1978; Westheimer, 1981), is readily explained by the use of a neural code equivalent to the centroid described here. This measure, which combines the responses of a number of neurons with overlapping receptive field profiles that have widths at halfheight of $4 \mathrm{~mm}$ or more (Dodson et al., 1998), is sensitive to changes in position smaller than the separation between adjacent receptive fields and smaller than the "size" of the receptive fields. Similar mechanisms have been proposed for hyperacuity in the electric fish (Heiligenberg, 1987).

A major strength of the modeling approach is shown by the results in Figure 10. Although innervation density is a property of the afferent fiber populations, its effect on resolution depends on noise levels and correlations that are primarily central in origin, a fact not usually acknowledged and not apparent without some 
sort of model. For neural codes that are entirely spatial in nature, such as the centroid of response, resolution increases with an increase in innervation density, regardless of the nature of the noise. Neural codes that reflect the average or total response of the neurons and are not spatial in nature, such as the measure used here for contact force, behave differently. Resolution improves with an increase in density if the noise for each afferent is independent of the noise on other afferents; however, when the noise is correlated, the effect of increasing density is diminished, consistent with the analysis of Zohary et al. (1994). From purely theoretical considerations, Johnson (1980b) pointed out that spatial and total response codes would differ in this regard. The situation is more complex for codes that contain elements of spatial codes and elements of total response codes, such as the measure used for the curvature of the stimulus.

Merkel endings are located at the bases of the glandular ridges (Halata, 1975), and there is a large variability in the pattern of skin ridges among human fingers. Thus, it is likely that the pattern of innervation varies considerably for different fingers; also, the loss of innervation with age is likely to be patchy. The neural measures used here, which are based on whole population responses, were not affected by nonuniformity in the pattern of innervation of the skin. In future, the model could be used to assess how critical it is that the brain has information about the position of the receptive field of each afferent.

\section{REFERENCES}

Abbott LF, Dayan P (1999) The effect of correlated variability on the accuracy of a population code. Neural Comput 11:91-101.

Aertsen A, Vaadia E, Abeles M, Ahissar E, Bergman H, Karmon B, Lavner Y, Margalit E, Nelken I, Rotter S (1991) Neural interactions in the frontal cortex of a behaving monkey: signs of dependence on stimulus context and behavioral state. J Hirnforsch 32:735-743.

Barlow HB (1972) Single units and sensation: a neuron doctrine for perceptual psychology? Perception 1:371-394.

Blake DT, Hsiao SS, Johnson KO (1997) Neural coding mechanisms in tactile pattern recognition: the relative contributions of slowly and rapidly adapting mechanoreceptors to perceived roughness. J Neurosci 17:7480-7489.

Brodie EE, Ross HE (1984) Sensorimotor mechanisms in weight discrimination. Percept Psychophys 36:477-481.

Cohen RH, Vierck CJ (1993) Population estimates for responses of cutaneous mechanoreceptors to a vertically indenting probe on the glabrous skin of monkeys. Exp Brain Res 94:105-119.

Croner L, Purpura K, Kaplan E (1993) Response variability in retinal ganglion cells of primates. Proc Natl Acad Sci USA 90:8128-8130.

Darian-Smith I, Kenins P (1980) Innervation density of mechanoreceptive fibres supplying glabrous skin of the monkey's index finger. J Physiol (Lond) 309:147-155.

Dean, AF (1981) The variability of discharge of simple cells in the cat striate cortex. Exp Brain Res 44:437-440.

Dodson MJ, Goodwin AW, Browning AS, Gehring HM (1998) Peripheral neural mechanisms determining the orientation of cylinders grasped by the digits. J Neurosci 18:521-530.

Edin BB, Essick GK, Trulsson M, Olsson KÅ (1995) Receptor encoding of moving tactile stimuli in humans. I. Temporal pattern of discharge of individual low-threshold mechanoreceptors. J Neurosci 15:830-847.

Edwards D, Purpura K, Kaplan E (1995) Contrast sensitivity and spatial frequency response of primate cortical neurons in and around the cytochrome oxidase blobs. Vision Res 35:1501-1523.

Fetz EE (1997) Temporal coding in neural populations? Science 278:1901-1902.

Gardner EP, Costanzo RM (1980) Spatial integration of multiple-point stimuli in primary somatosensory cortical receptive fields of alert monkeys. J Neurophysiol 43:420-443.

Gawne TJ, Richmond BJ (1993) How independent are the messages carried by adjacent inferior temporal cortical neurons? J Neurosci 13:2758-2771.
Georgopoulos AP, Schwartz AB, Kettner RE (1986) Neuronal population coding of movement direction. Science 233:1416-1419.

Gerstein GL, Aertsen AM (1985) Representation of cooperative firing activity among simultaneously recorded neurons. J Neurophysiol 54:1513-1528.

Gochin PM, Colombo M, Dorfman GA, Gerstein GL, Gross CG (1994) Neural ensemble coding in inferior temporal cortex. J Neurophysiol 71:2325-2337.

Goodwin AW, Wheat HE (1992) Magnitude estimation of contact force when objects with different shapes are applied passively to the fingerpad. Somatosens Mot Res 9:339-344.

Goodwin AW, Wheat HE (1999) Responses of slowly adapting cutaneous mechanoreceptive afferent fibres to three-dimensional tactile stimuli. In: Somatosensory processing: from single neuron to brain imaging (Rowe MJ, Iwamura Y, eds). London: Harwood.

Goodwin AW, John KT, Marceglia AH (1991) Tactile discrimination of curvature by humans using only cutaneous information from the fingerpads. Exp Brain Res 86:663-672.

Goodwin AW, Browning AS, Wheat HE (1995) Representation of curved surfaces in responses of mechanoreceptive afferent fibers innervating the monkey's fingerpad. J Neurosci 15:798-810.

Goodwin AW, Macefield VG, Bisley JW (1997) Encoding of object curvature by tactile afferents from human fingers. J Neurophysiol 78:2881-2888.

Halata Z (1975) The mechanoreceptors of the mammalian skin. Ultrastructure and morphological classification. Adv Anat Embryol Cell Biol 50:1-77.

Heggelund P, Albus K (1978) Response variability and orientation discrimination of single cells in striate cortex of cat. Exp Brain Res 32:197-211.

Heiligenberg W (1987) Central processing of sensory information in electric fish. J Comp Physiol [A] 161:621-631.

Johansson RS, Vallbo AB (1979) Tactile sensibility in the human hand: relative and absolute densities of four types of mechanoreceptive units in glabrous skin. J Physiol (Lond) 286:283-300.

Johnson KO (1974) Reconstruction of population response to a vibratory stimulus in quickly adapting mechanoreceptive afferent fiber population innervating glabrous skin of the monkey. J Neurophysiol 37:48-72.

Johnson KO (1980a) Sensory discrimination: decision process. J Neurophysiol 43:1771-1792.

Johnson KO (1980b) Sensory discrimination: neural processes preceding discrimination decision. J Neurophysiol 43:1793-1815.

Johnson KO, Darian-Smith I, LaMotte C, Johnson B, Oldfield S (1979) Coding for incremental changes in skin temperature by a population of warm fibres in the monkey: correlation with intensity discrimination in man. J Neurophysiol 42:1332-1353.

Khalsa PS, Friedman RM, Srinivasan MA, LaMotte RH (1998) Encoding of shape and orientation of objects indented into the monkey fingerpad by populations of slowly and rapidly adapting mechanoreceptors. J Neurophysiol 79:3238-3251.

Knibestol M (1975) Stimulus-response functions of slowly adapting mechanoreceptors in the human glabrous skin area. J Physiol (Lond) 245:63-80.

LaMotte RH, Srinivasan MA (1987a) Tactile discrimination of shape: responses of slowly adapting mechanoreceptive afferents to a step stroked across the monkey fingerpad. J Neurosci 7:1655-1671.

LaMotte RH, Srinivasan MA (1987b) Tactile discrimination of shape: responses of rapidly adapting mechanoreceptive afferents to a step stroked across the monkey fingerpad. J Neurosci 7:1672-1681.

LaMotte RH, Srinivasan MA (1996) Neural encoding of shape: Responses of cutaneous mechanoreceptors to a wavy surface stroked across the monkey fingerpad. J Neurophysiol 76:3787-3797.

LaMotte RH, Srinivasan MA, Lu C, Klusch-Petersen A (1994) Cutaneous neural codes for shape. Can J Physiol Pharmacol 72:498-505.

Lee D, Port NL, Kruse W, Georgopoulos AP (1998) Variability and correlated noise in the discharge of neurons in motor and parietal areas of the primate cortex. J Neurosci 18:1161-1170.

Lehky SR, Sejnowski TJ (1990) Neural network model of visual cortex for determining surface curvature from images of shaded surfaces. Proc R Soc Lond B Biol Sci 240:251-278.

Loomis JM, Collins CC (1978) Sensitivity to shifts of a point stimulus: an instance of tactile hyperacuity. Percept Psychophys 24:487-492.

Mountcastle VB, Reitboeck HJ, Poggio GF, Steinmetz MA (1991) Adaptation of the Reitboeck method of multiple microelectrode recording to the neocortex of the waking monkey. J Neurosci Methods 36:77-84. 
Paradiso MA (1988) A theory for the use of visual orientation information which exploits the columnar structure of striate cortex. Biol Cybern 58:35-49.

Phillips JR, Johansson RS, Johnson KO (1992) Responses of human mechanoreceptive afferents to embossed dot arrays scanned across fingerpad skin. J Neurosci 12:827-839.

Press WH, Flannery BP, Teukolsky SA, Vetterling WT (1986) Numerical recipes. The art of scientific computing. Cambridge, UK: Cambridge UP.

Ray RH, Doetsch GS (1990) Coding of stimulus location and intensity in populations of mechanosensitive nerve fibers of the raccoon. II. Acrossfiber response patterns. Brain Res Bull 25:533-550.

Rieke F, Warland D, De Ruyter Van Steveninck RR, Bialek W (1997) Spikes: exploring the neural code. Cambridge, MA: MIT.

Robinson DA (1992) Implications of neural networks for how we think about brain function. Behav Brain Sci 15:644-655.

Schiller PH, Finlay BL, Volman S (1976) Short-term response variability of monkey striate neurons. Brain Res 105:347-349.

Schreiner RC, Essick GK, Whitsel BL (1978) Variability in somatosensory cortical neuron discharge: effects on capacity to signal different stimulus conditions using a mean rate code. J Neurophysiol 41:338-349.

Shadlen MN, Britten KH, Newsome WT, Movshon JA (1996) A computational analysis of the relationship between neuronal and behavioral responses to visual motion. J Neurosci 16:1486-1510.

Snowden RJ, Treue S, Andersen RA (1992) The response of neurons in areas $\mathrm{V} 1$ and $\mathrm{MT}$ of the alert rhesus monkey to moving random dot patterns. Exp Brain Res 88:389-400.

Srinivasan MA, LaMotte RH (1987) Tactile discrimination of shape: responses of slowly and rapidly adapting mechanoreceptive afferents to a step indented into the monkey fingerpad. J Neurosci 7:1682-1697.

Tremblay F, Ageranioti-Bélanger SA, Chapman CE (1996) Cortical mechanisms underlying tactile discrimination in the monkey. I. Role of primary somatosensory cortex in passive texture discrimination. J Neurophysiol 76:3382-3403.

Vogels R, Spileers W, Orban GA (1989) The response variability of striate cortical neurons in the behaving monkey. Exp Brain Res 77:432-436.

Westheimer G (1981) Visual hyperacuity. Prog Sens Physiol 1:1-30.

Wheat HE, Goodwin AW, Browning AS (1995) Tactile resolution: peripheral neural mechanisms underlying the human capacity to determine positions of objects contacting the fingerpad. J Neurosci 15:5582-5595.

Whitsel BL, Schreiner RC, Essick GK (1977) An analysis of variability in somatosensory cortical neuron discharge. J Neurophysiol 40:589-607.

Whitsel BL, Dreyer DA, Hollins M (1978) Representation of moving stimuli by somatosensory neurons. Fed Proc 37:2223-2227.

Zhang KC, Ginzburg I, McNaughton BL, Sejnowski TJ (1998) Interpreting neuronal population activity by reconstruction: unified framework with application to hippocampal place cells. J Neurophysiol 79:1017-1044.

Zohary E, Shadlen MN, Newsome WT (1994) Correlated neuronal discharge rate and its implications for psychophysical performance. Nature 370:140-143. 